\title{
Incapacidade cervical está relacionada à presença de aura e frequência de crises de migrânea
}

\author{
Gabriella de Almeida Tolentino ${ }^{1}$ (D) , Carina Ferreira Pinheiro' ${ }^{1 D}$, Lidiane Lima Florencio ${ }^{2}$ (D),

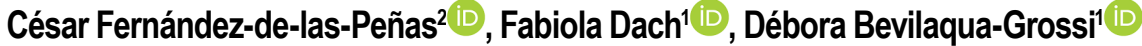

'Faculdade de Medicina de Ribeirão Preto, Universidade de São Paulo, Ribeirão Preto, São Paulo, Brasil. E-mail:

2Universidade Rey Juan Carlos, Móstoles, Madrid, Espanha

\section{Introdução}

A cervicalgia é altamente prevalente em migranosos, e um potencial contribuinte para a incapacidade relacionada à cefaleia. Além disso, indivíduos com migrânea crônica apresentam maior incapacidade cervical, entretanto não é conhecido se esta relação também se manifesta em indivíduos com aura. $\mathrm{O}$ objetivo deste estudo foi avaliar as características da cervicalgia em mulheres com migrânea com e sem aura e migrânea crônica.

\section{Métodos}

Este estudo transversal incluiu 102 mulheres entre 18 e 55 anos de idade. Destas, 70 foram divididas em três grupos de acordo com o diagnóstico: migrânea sem aura (MSA, $n=22)$, migrânea com aura (MCA, $n=17$ ) e migrânea crônica ( $M C, n=31)$. Mulheres sem cefaleia compuseram o grupo controle ( $G C, n=32)$. As participantes foram questionadas quanto às características clínicas da cefaleia e da cervicalgia. As voluntárias com relato de cervicalgia completaram o questionário Neck Disability Index, para avaliação da incapacidade relacionada à dor cervical. O estudo foi aprovado pelo comitê de ética e pesquisa (CAAE 59620716.2.0000.5440).

\section{Resultados}

As variáveis de desfecho foram comparadas entre os grupos por meio de ANOVA one-way e Teste de Qui-quadrado $(p<0,05)$. O relato de cervicalgia foi maior nos grupos migranosos (GC 25\%, MSA 50\%, MCA 100\% e MC 81\%, $p<0,001)$. Quanto às características da cervicalgia, o grupo $M C$ apresentou maior frequência de cervicalgia $(21,1$ dias/mês, DP 8, 1), comparado ao grupo MSA (9,2 dias mês, DP 10,5; $p=0,003)$. Além disso, maior incapacidade cervical foi observada no grupo MC comparado ao MSA e GC, e no grupo MCA comparado ao GC (GC 5,4 pontos, DP 5,4; MSA 9,9 pontos, DP 4,2; MCA 14,8 pontos, DP 6, 1; MC 16,5 pontos, DP 7,0; $p<0.001$ ).

\section{Conclusão}

Embora a frequência de cervicalgia seja alta nos pacientes com migrânea crônica, a prevalência de cervicalgia e a incapacidade cervical são maiores em migranosos com aura e migrânea crônica.

Palavras-chave: Transtornos da Enxaqueca, Enxaqueca, com aura, Cervicalgia. 